\title{
Schistosome-induced cholangiocyte proliferation and osteopontin secretion correlate with fibrosis and portal hypertension in human and murine schistosomiasis mansoni
}

\author{
Thiago A. Pereira*†, Wing-Kin Synキ§, Mariana V. Machado*, Paula V. Vidigal\|, Vivian Resende\|, \\ Izabela Voieta\|, Guanhua Xie*, Alba Otoni\|, Márcia M. Souza†, Elisângela T. Santos†, Isaac S. Chan*, \\ Guilherme V.M. Trindade\|, Steve S. Choi*, Rafal P. Witekף, Fausto E. Pereira**, William E. Secor††, \\ Zilton A. Andrade†, José Roberto Lambertucci\| and Anna Mae Diehl* \\ *Division of Gastroenterology, Department of Medicine, Duke University Medical Center, Durham, NC 27710, U.S.A. \\ †Laboratório de Patologia Experimental, Centro de Pesquisas Gonçalo Moniz/FIOCRUZ, Salvador, BA 40296-710, Brazil \\ \#Liver Regeneration and Repair Research Group, Institute of Hepatology, Foundation for Liver Research, London WC1E 6HX, U.K. \\ $\S$ Department of Surgery, Loyola University, Chicago, Maywood, IL 60153, U.S.A. \\ \|Faculdade de Medicina, Universidade Federal de Minas Gerais, Belo Horizonte, MG 30130-100, Brazil \\ ףLife Technologies, Frederick, MD 21704, U.S.A. \\ **Núcleo de Doenças Infecciosas, Universidade Federal do Espírito Santo, Vitória, ES 29040-091, Brazil \\ + †enters for Disease Control and Prevention, Atlanta, GA 30329, U.S.A.
}

\begin{abstract}
Schistosomiasis is a major cause of portal hypertension worldwide. It associates with portal fibrosis that develops during chronic infection. The mechanisms by which the pathogen evokes these host responses remain unclear. We evaluated the hypothesis that schistosome eggs release factors that directly stimulate liver cells to produce osteopontin (OPN), a pro-fibrogenic protein that stimulates hepatic stellate cells to become myofibroblasts. We also investigated the utility of OPN as a biomarker of fibrosis and/or severity of portal hypertension. Cultured cholangiocytes, Kupffer cells and hepatic stellate cells were treated with soluble egg antigen (SEA); OPN production was quantified by quantitative reverse transcriptase polymerase chain reaction (qRTPCR) and ELISA; cell proliferation was assessed by BrdU (5-bromo-2'-deoxyuridine). Mice were infected with Schistosoma mansoni for 6 or 16 weeks to cause early or advanced fibrosis. Liver OPN was evaluated by qRTPCR and immunohistochemistry (IHC) and correlated with liver fibrosis and serum OPN. Livers from patients with schistosomiasis mansoni (early fibrosis $n=15$; advanced fibrosis $n=72)$ or healthy adults $(n=22)$ were immunostained for OPN and fibrosis markers. Results were correlated with plasma OPN levels and splenic vein pressures. SEA-induced cholangiocyte proliferation and OPN secretion $(P<0.001$ compared with controls). Cholangiocytes were OPN $(+)$ in Schistosoma-infected mice and humans. Liver and serum OPN levels correlated with fibrosis stage (mice: $r=$ 0.861; human $r=0.672, P=0.0001$ ) and myofibroblast accumulation (mice: $r=0.800$; human: $r=0.761$, $P=0.0001)$. Numbers of OPN $(+)$ bile ductules strongly correlated with splenic vein pressure $(r=0.778$; $P=0.001)$. S. mansoni egg antigens stimulate cholangiocyte proliferation and OPN secretion. OPN levels in liver and blood correlate with fibrosis stage and portal hypertension severity.
\end{abstract}

Key words: cholangiocyte, ductular proliferation, osteopontin, portal hypertension, Schistosomiasis mansoni, Symmers' fibrosis.

\section{INTRODUCTION}

Schistosomiasis or bilharzia is an infectious tropical disease caused by trematode worms of the genus Schistosoma that affects over 440 million people from 76 countries and territories [1,2]. Chronic infection with Schistosoma mansoni or Schistosoma japonicum, the major Schistosoma species that affect humans, can cause severe fibrosis and associated portal hypertension, leading

Abbreviations: BrdU, 5-bromo-2'-deoxyuridine; FFPE, formalin-fixed, paraffin-embedded; HI, hepatointestinal; HS, hepatosplenic; HSC, hepatic stellate cell; IHC, immunohistochemistry; K19, keratin 19; LPS, lipopolysaccharide; OPN, osteopontin; qRTPCR, quantitative reverse transcriptase polymerase chain reaction; SEA, soluble egg antigen; $\alpha$ SMA, $\alpha$-smooth muscle actin; WHO, World Health Organization.

Correspondence: Dr Anna Mae Diehl (email diehl004@mc.duke.edu). 
to disease-related morbidity and mortality [1]. The chronic phase of schistosomiasis has two main disease forms: hepatointestinal (HI) and hepatosplenic (HS) [3]. Eggs deposited in the mesenteric veins are carried into the microvasculature of the liver portal system and induce a granulomatous reaction that evolves to portal fibrosis [4]. The majority of individuals from endemic areas who become infected with $S$. mansoni or S. japonicum develop HI schistosomiasis [3]. This is a mild disease in which few hepatic granulomas form within small peripheral branches of the portal vein, inducing minimal hepatic fibrosis which is asymptomatic in most cases $[4,5]$. However, a small percentage of infected individuals (4-10\%) develop a severe form of the disease in which numerous granulomas form within large branches of the portal vasculature [4-6]. This incites vascular proliferation, characteristic pipe-stem-like fibrotic expansion of most portal tracts (dubbed Symmers' fibrosis), splenomegaly and portal hypertension [4-6]. Fortunately, the fibrotic response is restricted to the portal area; there is minimal damage to hepatocytes; the lobular architecture of the liver is maintained; and liver function is well preserved [4-6].

Why only a small percentage of infected individuals develop HS schistosomiasis is still an open question [3,7]. Worm load, infection during infancy and multiple re-infections are decisive in the outcome of disease, but other factors such as host genetic background, parasite strains and parasite-host interactions are probably involved [4,5,7]. Although it is well-established that the granulomatous reaction around the schistosome egg induces fibrosis, the mechanisms driving fibrogenesis have not been fully elucidated [4,5,7]. Recently the Hedgehog pathway was identified as one of the major regulators of schistosomiasis-related fibrosis and vascular remodelling [8]. This pathway regulates the activation of hepatic stellate cells (HSCs), the major matrix-producing liver cell type and is involved in several fibrotic diseases of the liver [9]. In schistosomiasis mansoni, egg antigens stimulate liver macrophages to produce Hedgehog ligands, which promote alternative activation of macrophages, activation of HSCs and angiogenesis [8].

The pro-fibrogenic cytokine osteopontin (OPN) is one of the target genes of the Hedgehog pathway [10]. Syn et al. [10] elegantly demonstrated that this molecule is the effective mediator of the pro-fibrogenic effects induced by the Hedgehog pathway. They showed that OPN regulates HSC activation and induces collagen I synthesis [10]. Neutralization of OPN inhibited the myofibroblastic transdifferentiation of these cells [10]. Circulating OPN levels correlate with fibrosis stage in several types of liver disease, such as alcoholic [11,12] and non-alcoholic [13] steatohepatitis, viral hepatitis B [14] and C [15] and congenital biliary disease [16]. OPN has also been described as a potential biomarker for liver cancer [17]. In addition to its direct actions on HSCs, OPN plays a role in granulomatous reactions by regulating the recruitment of monocytes/macrophages [18]. Previously it was demonstrated that OPN correlates with fibrogenesis in experimental schistosomiasis japonica [19] and neutralization of OPN reduces fibrosis in this animal model of the human disease [20].

OPN is produced by many types of cells [21], but reactiveappearing bile-duct-like cells (cholangiocytes) are a major source of OPN in many liver diseases [22,23]. These cholangiocytes also produce Hedgehog ligands and other fibrogenic factors [9]. In addition, they secrete a series of chemokines that recruit immune cells to the liver [24]. Finally, some reactive cholangiocytes may be able to undergo an epithelial-to-mesenchymal transition to generate myofibroblasts during liver injury [25]. Although it is well known that reactive-appearing duct-like cells accumulate and form bile ductules in and around portal tracts during progression of human and experimental schistosomiasis, this ductular reaction is thought to be a non-specific phenomenon that has little consequence for disease progression [26-29].

In the present study, we demonstrated for the first time that soluble egg antigen (SEA) induces cholangiocyte proliferation and that OPN secretion. The number of OPN-expressing bile ductules and the circulating OPN levels correlated with collagen deposition and myofibroblast activation in murine and human schistosomiasis, suggesting that OPN might be useful as a potential biomarker of schistosomiasis severity. We also demonstrated that the number of OPN $(+)$ bile ducts correlate with the severity of portal hypertension in humans and thus propose a novel mechanistic link between the OPN-producing ductular reaction and portal hypertension pathogenesis.

\section{MATERIALS AND METHODS}

\section{Patients}

A total of 72 formalin-fixed, paraffin-embedded (FFPE) wedge liver biopsies from patients with HS schistosomiasis mansoni (Symmers' fibrosis and portal hypertension) collected during splenectomy and diagnosed at the University Hospital of Universidade Federal de Minas Gerais and archived in the Anatomical Pathology Department (between 1998 and 2011) were included in the present study. FFPE needle liver biopsies from patients with HI schistosomiasis (liver granulomas without Symmers' fibrosis, $n=15)$ and from liver transplant donors were used as controls $(n=22)$. A subgroup of patients/controls were examined by ultrasound and the degree of liver fibrosis were evaluated according to the World Health Organization (WHO) Niamey-Belo Horizonte Protocol $(n=67)$. Splenic vein pressures were studied in 15 patients with HS schistosomiasis at the time of splenectomy. A total of 87 plasma samples from patients with schistosomiasis (HS: $n=39$; HI: $n=27$ ) diagnosed at the Tropical Diseases Outpatient Clinic of the UFMG Hospital and from uninfected controls $(n=21)$ were also included. Demographic data on patients and controls are summarized in Table 1 . The present study was conducted in accordance with the Declaration of Helsinki (2013) of the World Medical Association and was approved by the Ethics Committee of UFMG (Protocol ETIC 204/06). Informed consent was obtained from participating subjects.

\section{Animal studies}

Female Swiss Webster outbred mice were infected with 50 cercariae of S. mansoni (Feira de Santana strain, CPqGM/FIOCRUZ) for 6 weeks (early fibrosis group, $n=5$ ) and 16 weeks (advanced fibrosis group, $n=7$ ). Uninfected, age- and strain-matched 


\begin{tabular}{lrl}
\hline $\begin{array}{l}\text { Table } 1 \\
\text { schistosomiasis mansoni and controls included in the present } \\
\text { study }\end{array}$ & $\begin{array}{c}\text { The demographic data of the selected patients with } \\
\text { Age }\end{array}$ & $\begin{array}{l}\text { Gender } \\
\text { (M, male; F, female) }\end{array}$ \\
\hline & (mean \pm S.D.) & \\
& & $16 \mathrm{M} / 6 \mathrm{~F}$ \\
\hline FFPE liver biopsies & $26 \pm 10.64$ & $\begin{array}{l}11 \mathrm{M} / 4 \mathrm{~F} \\
\text { Healthy individuals }\end{array}$ \\
$\begin{array}{l}\text { HI schistosomiasis } \\
\text { HS schistosomiasis }\end{array}$ & $33.9 \pm 10.84 \pm 13.72$ & $50 \mathrm{M} / 22 \mathrm{~F}$ \\
$\begin{array}{l}\text { Plasma samples } \\
\text { Healthy individuals }\end{array}$ & $24 \pm 8$ & $14 \mathrm{M} / 7 \mathrm{~F}$ \\
HI schistosomiasis & $36.75 \pm 12.93$ & $15 \mathrm{M} / 12 \mathrm{~F}$ \\
HS schistosomiasis & $36.7 \pm 11.67$ & $30 \mathrm{M} / 9 \mathrm{~F}$ \\
\hline
\end{tabular}

animals were used as controls $(n=7)$. Liver tissue and serum were collected for analysis. The present study was approved (Protocol 003/2010) by the Ethical Committee for Animal Research of CPqGM/FIOCRUZ.

\section{Soluble egg antigen preparation, endotoxin testing and cell culture studies}

The SEA was prepared at Centers for Disease Control and Prevention (CDC). The amount of Gram negative bacterial endotoxin present in the SEA preparation was quantified using the end-point chromogenic limulus amebocyte lysate assay (Lonza). Mouse cholangiocyte line 603B, primary human cholangiocytes (from Life Technologies), normal human cholangiocyte cell line H69 [a gift from Professor G.J. Gores (Mayo Clinic, Rochester, MN, U.S.A.) and Professor G.D. Alpini (Texas A\&M University System Health Science Center, Temple, TX, U.S.A.)] and clonally derived rat HSC line 8B [a gift from Professor M. Rojkind (George Washington University, Washington, DC, U.S.A., in memoriam)] were incubated with $10 \mu \mathrm{g} / \mathrm{ml} \mathrm{SEA}$ or $0.0001 \mu \mathrm{g} / \mathrm{ml}$ LPS (lipopolysaccharide; control, same amount of endotoxin present in the SEA preparation) for 2, 6, 12 and $24 \mathrm{~h}$. RNA and conditioned medium were collected for analysis. Cell proliferation was assessed by 5-bromo-2'-deoxyuridine (BrdU) incorporation (Cell Signaling Technology) after $24 \mathrm{~h}$ of SEA or control incubation.

\section{OPN ELISA}

OPN was quantified in the plasma (humans), serum (mice) or conditioned medium (cells) using OPN Quantikine ELISA kit (R\&D Systems) according to the manufacturer's protocol.

\section{RNA analysis}

RNA was extracted using RNeasy mini kit (Qiagen) according to the manufacture's protocol. Reverse transcription was performed using the First Strand Superscript III kit (Life Technologies) using the random hexamers protocol. OPN mRNA expression was evaluated by real-time PCR (SYBR Green). Each sample was analysed in duplicate and target gene levels in treated cells are shown as a ratio to levels detected in corresponding control samples, according to the $\Delta \Delta C_{\mathrm{T}}$ method, relative to the housekeeping gene (S9). The following primer probes were used: mouse/rat S9 forward 3'-GAC TCC GGA ACA AAC GTG AGG T-5', mouse/rat
S9 reverse 3'-CTT CAT CTT GCC CTC GTC CA-5', mouse OPN forward 3'-CTC CAT CGT CAT CAT CAT CG-5', mouse OPN reverse $3^{\prime}$-TGC ACC CAG ATC CTA TAG CC- $5^{\prime}$, human $\beta$-actin forward $3^{\prime}$-TGG CAT CCA CGA AAC TAC CT-5', human $\beta$-actin reverse $3^{\prime}$-ACG GAG TAC TTG CGC TCA G- $5^{\prime}$, human OPN forward $3^{\prime}$-TGA AAT TCA TGG CTA TGG AA- $5^{\prime}$, human OPN reverse $3^{\prime}$-TGA AAC GAG TCA GCT GGA TG-5', rat OPN forward 3'-ATG GCT TTC ATT GGA GTT GC-5' , rat OPN reverse 3'- GAG GAG AAG GCG CAT TAC AG-5', rat $\alpha$ smooth muscle actin ( $\alpha$ SMA) forward $3^{\prime}$-GTG GAT CAC CAA GCA GGA GGA GT- $5^{\prime}$, rat $\alpha$ SMA reverse $3^{\prime}$ - CAT AGC ACG ATG GTC GAT TG-5'.

\section{Immunohistochemisty}

Liver sections were stained with H\&E (haematoxylin and eosin) for general histology and Picro-Sirius Red for fibrosis assessment. Immunohistochemistry (IHC) analysis was performed to evaluate the expression of $\alpha$ SMA (myofibroblast marker), desmin HSC marker), OPN, CD44 (OPN receptor), keratin 19 (K19, cholangiocyte marker), and CD68 and F4-80 (macrophage markers). The antigen retrieval and dilutions for the antibodies used are provided in Supplementary Online Data. Picro Sirius Red and $\alpha$ SMA staining was quantified in ten $\times 100$ fields/sample by computer-assisted morphometry using MetaMorph (Universal Imaging Corp.). OPN (+) bile ducts were counted in ten $\times 200$ fields/sample by three independent observers. To identify the types of cells that produce or respond to OPN, double IHC was performed using the chromagen DAB (3,3'-diaminobenzidine) for OPN or CD44 and the chromagen Vina Green for K19, CD68, F4-80, $\alpha$ SMA or desmin.

\section{Statistical analysis}

Results are expressed as means \pm S.E.M. (for normal distribution variables) or as medians (for non-normal distribution variables). Comparisons between groups were performed using the oneway ANOVA and Student's $t$ test (parametric) or Kruskal-Wallis one-way ANOVA and Mann-Whitney $U$ test (non-parametric). Significance was accepted at the 0.05 level; Bonferroni correction was applied when comparing more than two groups. Pearson's correlation $(r)$ and linear regression analysis $\left(r^{2}\right)$ or Spearman's correlation $\left(r_{\mathrm{s}}\right)$ were used. All statistical analyses were performed using SPSS Statistics 22 (IBM) and Prism 6 (GraphPad).

\section{RESULTS}

\section{SEA stimulates cholangiocyte proliferation and osteopontin production}

To investigate whether $S$. mansoni directly stimulates cholangiocyte proliferation, cultured murine or human cholangiocytes were incubated with $10 \mu \mathrm{g} / \mathrm{ml} \mathrm{SEA} \mathrm{or} \mathrm{vehicle} \mathrm{(control).} \mathrm{SEA} \mathrm{directly}$ induced cholangiocyte proliferation and up-regulated expression of OPN mRNA (Figures 1A-1D). The effects of SEA on cholangiocyte OPN production occurred very rapidly. Incubation with SEA stimulated the secretion of OPN within $2 \mathrm{~h}$ of exposure (Figure 1C). We next evaluated whether cholangiocytes from human and murine schistosomiasis mansoni expressed OPN. 


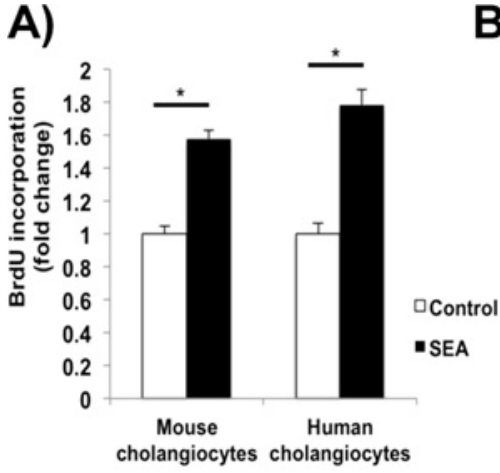

D)

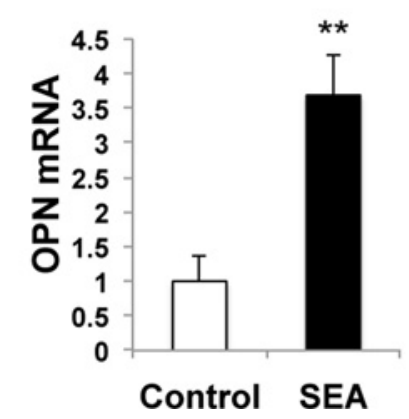

E)

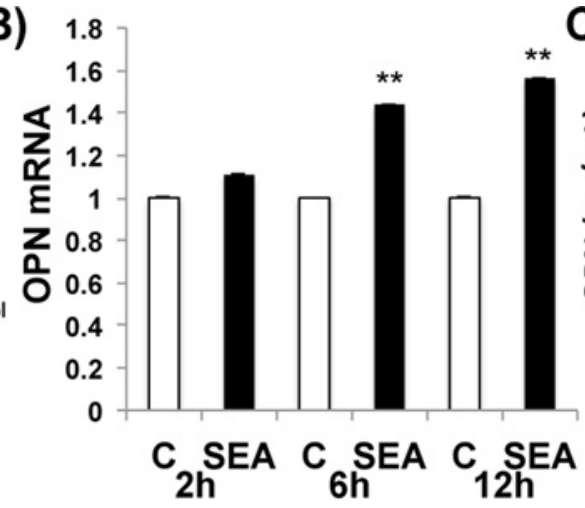

C) 350

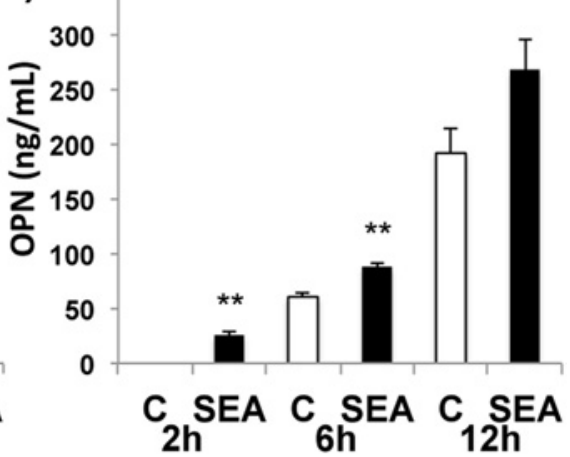

Figure 1 SEA induces cholangiocyte proliferation and OPN secretion

(A) Cellular proliferation assay (BrdU incorporation). Mouse cholangiocyte cell line 603B and primary human cholangiocytes were treated with $10 \mu \mathrm{g} / \mathrm{ml} \mathrm{S}$. mansoni SEA or $0.0001 \mu \mathrm{g} / \mathrm{ml}$ LPS (control) for $24 \mathrm{~h}$. (B) OPN mRNA expression in mouse cholangiocytes (603B) treated with SEA or control for 2, 6 and $12 \mathrm{~h}$. Results were normalized by mRNA expression at each time point control; means \pm S.E.M. are displayed. (C) Quantification of OPN in the conditioned medium of murine cholangiocytes treated with SEA or control (ELISA). Medians are displayed. No difference was observed at $24 \mathrm{~h}$ in (B) and (C). (D) OPN mRNA expression in normal human cholangiocyte cell line $\mathrm{H} 69$ treated with SEA or control for $12 \mathrm{~h}$. No significant differences were observed at 2, 6 and $24 \mathrm{~h}$. Results were normalized by mRNA expression at each time point control; means \pm S.E.M. are displayed. (E) Double immunostaining for OPN (brown) and the cholangiocyte marker K19 (green) in human (left) and murine (right) schistosomiasis. Final magnification $\times 200$. $* P<0.05 ; * * P<0.005$.

Double IHC for OPN and K19 (a cholangiocyte marker) confirmed that the majority of $\mathrm{K} 19(+)$ cholangiocytes were OPN $(+)$ (Figure 1E). These results demonstrate that the bile ductular reaction observed in human and murine schistosomiasis is induced by egg-derived factors that are released during $S$. mansoni infection. Moreover, the findings suggest that that this ductular response drives schistosomiasis-related liver fibrosis and identify a likely mechanism, namely egg-induced up-regulation of OPN production by cholangiocytes.

\section{OPN is up-regulated in murine schistosomiasis and correlates with fibrogenesis}

To determine whether OPN production also increased during in vivo schistosoma infection, a mouse model of schistosomiasis was studied. OPN-producing liver cells accumulated in murine schistosomiasis. Furthermore, the number of OPN-producing cells correlated with fibrosis severity, being highest in mice with the most severe liver fibrosis (Figures 2A-2C). Serum OPN levels were similarly elevated in schistosomiasis, especially in mice with advanced diseases, suggesting its utility as a potential noninvasive marker of liver fibrosis (Figure 2D).
There was a significant increase in the number of OPN $(+)$ bile ductules in mice with advanced fibrosis (16 weeks post-infection) compared with mice with low fibrosis (6 weeks post-infection) or uninfected controls (Figure 2A). Importantly, the number OPN $(+)$ bile ductules and serum levels of OPN strongly correlated with the intensity of myofibroblast accumulation and severity of collagen deposition (Figure 2E). This finding suggests that the ductular reaction is a major source of OPN which drives fibrogenesis in murine schistosomiasis mansoni.

\section{OPN is up-regulated in human schistosomiasis and correlates with fibrogenesis}

Because neither cholangiocytes nor OPN had been previously implicated in the pathogenesis of human HS schistosomiasis, we felt that examination of tissue and blood samples from infected humans was essential. Similar to the animal model, patients with HS schistosomiasis were found to have increased number of OPN (+) bile ductules and circulating OPN levels compared with patients with HI schistosomiasis or uninfected controls (Figures $3 \mathrm{~A}-3 \mathrm{D}$ ). HI patients are usually asymptomatic and have a granulomatous reaction in the liver, but they are unlikely to 

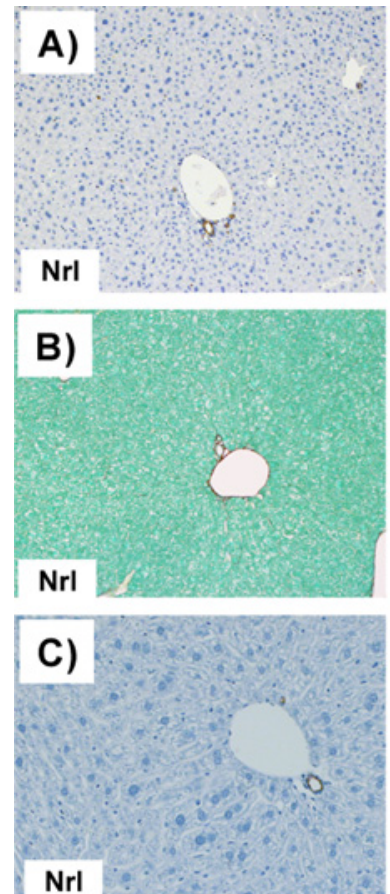

D)

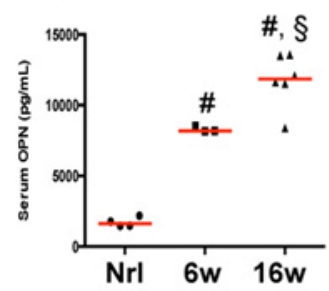

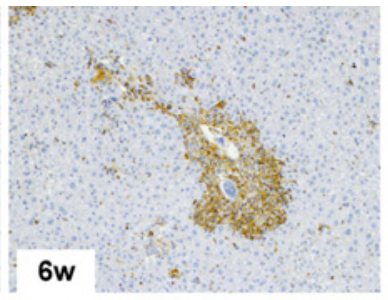
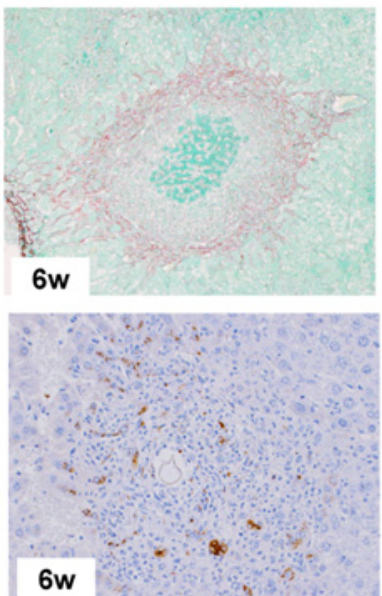

E)
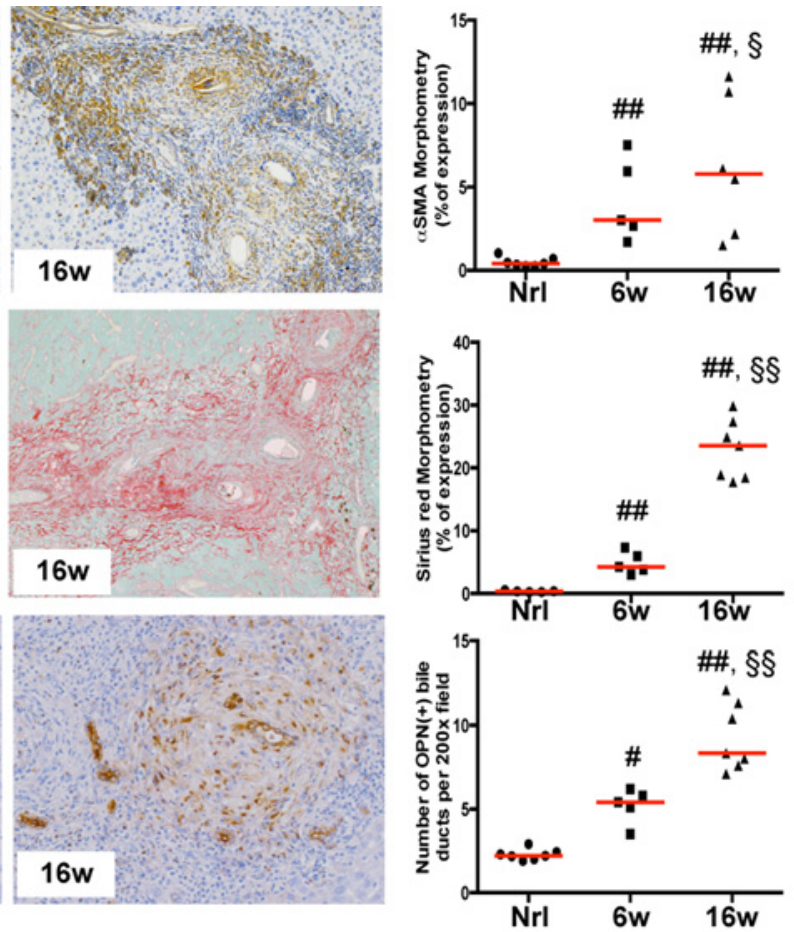

Figure 2 OPN is up-regulated in murine schistosomiasis and correlates with fibrogenesis

(A-C) $\alpha$ SMA immunostaining (A), Picro Sirius Red staining (B) and OPN immunostaining (C) on liver sections from a representative uninfected control mouse (left), from a representative mouse 6 weeks post-infection (low fibrosis; middle) and from a representative mouse 16 weeks post-infection (severe fibrosis; right). Final magnification $\times 100(\mathbf{A}$ and $\mathbf{B})$ or $\times 200$ (C). (D) Serum OPN levels in murine schistosomiasis (ELISA; normal (nrl) $n=4,6$ weeks $n=3,16$ weeks $n=6$ ). Medians are displayed; $\# P<0.05, \# \# P<0.005$ compared with $\mathrm{nrl}$; $\S P<0.05, \S \S P<0.005$ compared with 6 weeks. (E) Pearson's correlation and linear regression showing strong correlation between liver/blood OPN levels and fibrogenesis.

develop Symmers' fibrosis and related portal hypertension. Our results identify a likely explanation for the lack of disease progression, namely patients with HI schistosomiasis produce lower levels of OPN, a potent pro-fibrogenic factor.

Most of the HS patients we studied mainly expressed OPN in bile ductules, although a minority who were still in the active inflammatory stage of infection also expressed OPN in the granulomatous reaction (similar to $\mathrm{HI}$ patients and mice with schistosomiasis). The number of OPN (+) bile ductules strongly correlated with myofibroblast accumulation and the severity of collagen deposition (Figure 3E). Although paired plasma and tissue samples were available for very few patients with HS schistosomiasis, liver fibrosis severity had been staged according to the WHO ultrasound protocol (Nimey-Belo Horiorizonte) in most subjects. We observed a strong correlation between the degree of fibrosis staged by ultrasound, the number of OPN $(+)$ reactive bile ductules and circulating (plasma) OPN levels, suggesting that the latter may be a novel blood test to efficiently screen populations who are at high risk for severe schistosomiasis.

\section{OPN correlates with the degree of portal hypertension in human schistosomiasis}

Portal hypertension and its complications are the hallmark of HS schistosomiasis and responsible for the mortality associated with this life-threatening disease. The observation that patients with HI schistosomiasis (without portal hypertension) have lower serum levels of OPN and fewer OPN (+) reactive bile ducts than patients with HS schistosomiasis (with severe portal hypertension) suggested that this molecule may be indicative not only of liver fibrosis, but also of portal hypertension. Indeed, we observed a positive strong association between the number of OPN $(+)$ bile ductules and the degree of portal hypertension in a subgroup of HS schistosomiasis patients $(n=15)$ who had splenic venous pressure measured at the time of splenectomy (Figure 4). 

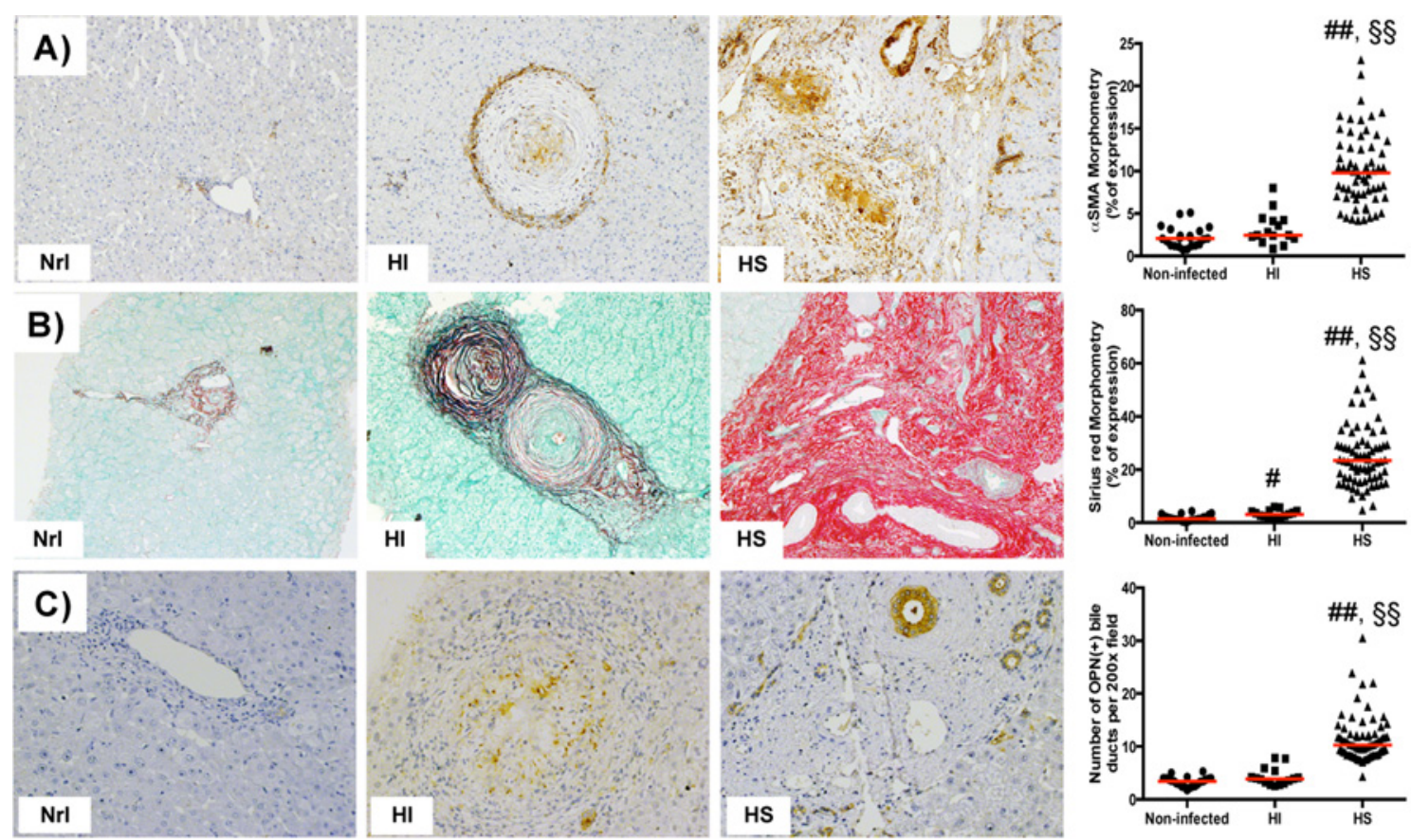

D)

E)
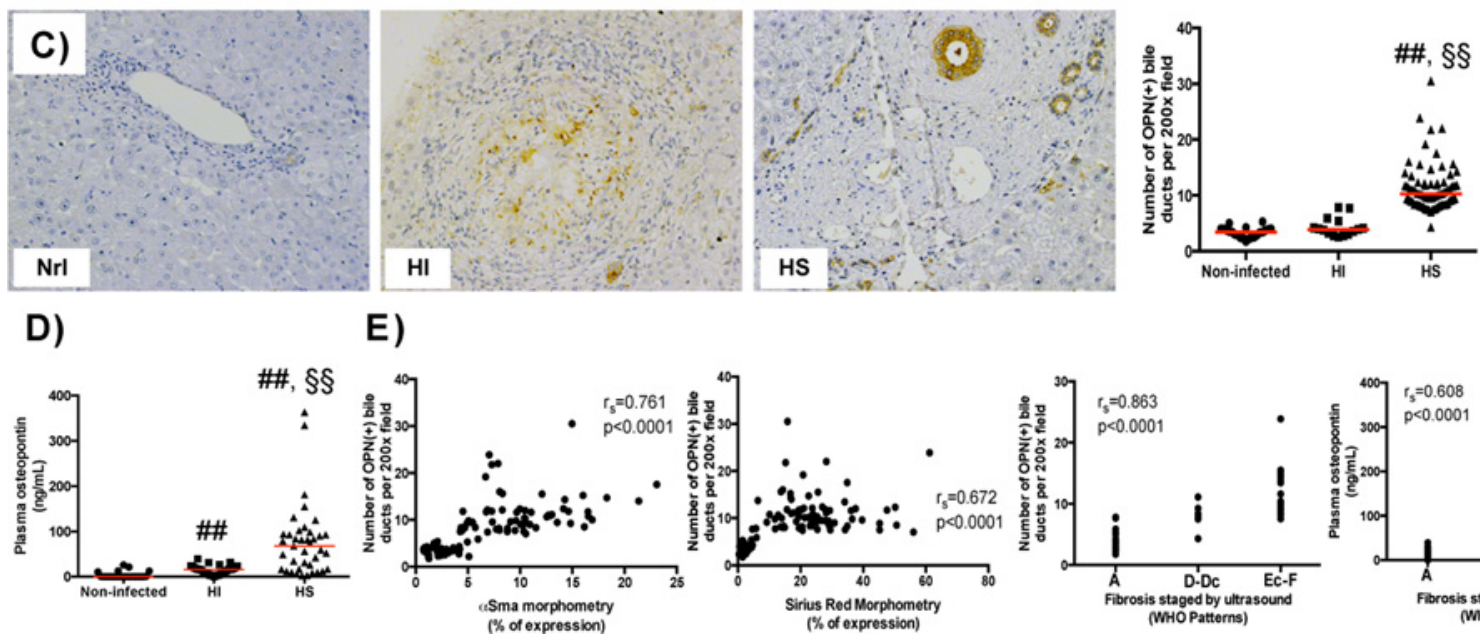

Figure 3 OPN is up-regulated in human schistosomiasis and correlates with fibrogenesis

(A-C) $\alpha$ SMA immunostaining (A), Picro Sirius Red staining (B) and OPN immunostaining (C) on liver sections from a representative uninfected healthy individual (left), from a representative patient with $\mathrm{HI}$ schistosomiasis (middle) and from a representative HS patient (right). Final magnification $\times 100($ A and B) or $\times 200$ (C). (D) Plasma OPN levels in human schistosomiasis (ELISA). Medians are displayed; $\# P<0.05, \# \# P<0.005$ compared with uninfected controls (nrl); $\S \S P<0.005$ compared with HI. (E) Spearman's correlation $\left(r_{\mathrm{s}}\right)$ showing strong correlation between liver/blood OPN levels and fibrogenesis.

Unfortunately plasma samples were not available from these patients. Hence, further studies are necessary to determine whether circulating OPN levels might be used as a non-invasive biomarker for portal hypertension severity in human schistosomiasis mansoni.

\section{Macrophages, myofibroblasts and HSCs located within granulomas also produce and respond to OPN}

As described above, cholangiocytes are the major source of OPN in advanced schistosomiasis mansoni (i.e. fewer granulomas, larger ductular reaction, more fibrosis). By contrast, during the early phase of the disease (i.e. more granulomas, smaller ductular reaction and less fibrosis), OPN (+) cells are located predominantly within granulomas. Using double IHC, we demonstrated that OPN (+) cells were F4/80 (+) or CD68 (+) and $\alpha$ SMA (+) or desmin $(+)$, therefore confirming that macrophages and myofibroblasts/ HSCs within granulomas (in mice and humans) are also capable of producing OPN (Figures 5A and 5B, left panels). Since our patient cohort was enriched with individuals who had advanced liver fibrosis, liver granulomas and granulomaassociated OPN (+) cells were not as frequent in human HS schistosomiasis as in the mouse model.

Since macrophages and HSC/myofibroblasts expressed OPN in vivo, we next investigated whether SEA could directly induce OPN in cultured cells. The addition of SEA led to a 7-fold increase in primary mouse Kupffer cells and a 2.5-fold increase in rat HSC 8B (Supplementary Figure S1). SEA also activated HSC and up-regulated the key HSC activation marker $\alpha$ SMA. We further investigated whether cells within the granulomatous reaction and/or fibrous septa might be responsive to OPN. In order to identify individual cells, double IHC was performed using markers for macrophages (F4/80 or CD68) or myofibroblasts/HSC ( $\alpha$ SMA and desmin) and CD44 (a putative OPN receptor). Granulomas and fibrotic portal tracts were enriched with CD44 (+) macrophages and myofibroblasts/HSCs (Figures 5A- 


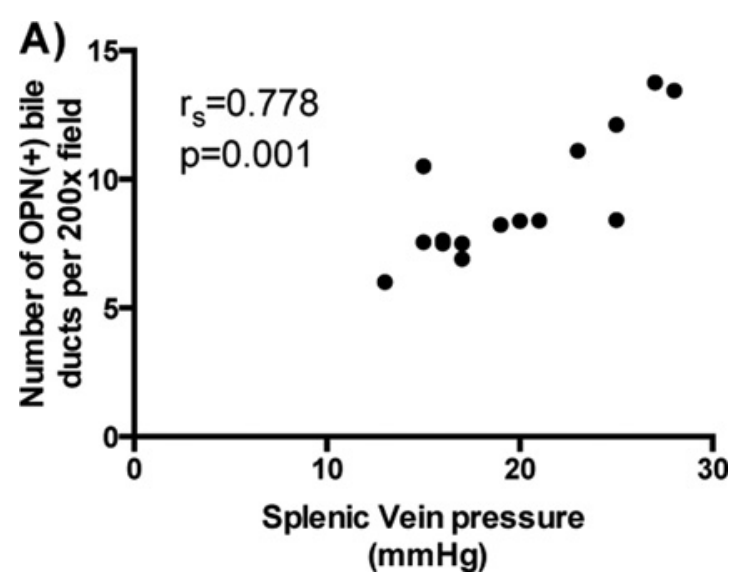

Figure 4 OPN correlates with severity of portal hypertension in human schistosomiasis

(A) Spearman's correlation $\left(r_{\mathrm{s}}\right)$ showing a strong significant correlation between the number of OPN $(+)$ bile ducts and the severity of portal hypertension in a subgroup of patients with HS schistosomiasis $(n=15)$.

$5 \mathrm{~B}$, right panels). The aggregate data reveal a dynamic interactive network of OPN-producing and OPN-responding cells in schistosoma infected liver.

In summary, these results identify a novel mechanism by which $S$. mansoni could promote liver fibrosis; egg antigens directly induce cholangiocytes, Kupffer cells and HSC to secrete
OPN. OPN in turn, bind to OPN-responsive cells to perpetuate the fibrogenic response. Furthermore, because OPN expression generally correlates with fibrosis severity across the spectrum of schistosomal liver disease, OPN is an attractive therapeutic target to prevent/reverse schistosomiasis mansoni fibrosis.

\section{DISCUSSION}

We discovered that soluble factors released by schistosoma eggs directly stimulate cholangiocytes to proliferate and produce OPN, a major pro-fibrogenic factor. We also observed that egg antigens could induce the production of OPN in Kupffer cells and HSCs, thereby increasing the total amount of OPN within granulomas and fibrous septa. Furthermore, we demonstrated that the number of OPN-expressing reactive-appearing bile ducts and the severity of liver fibrosis strictly correlated with each other in both murine models and humans with schistosomiasis mansoni. Vianna et al. [29] were the first to report that patients with HS schistosomiasis exhibit bile duct alterations, such as biliary hyperplasia, degeneration, proliferation and periductular fibrosis, but had concluded that those modifications were non-specific and irrelevant for the evolution of schistosomiasis fibrosis [26-29]. Our data, however, disprove that initial interpretation by showing that the bile duct reaction observed in schistosomiasis is specifically induced by Schistosoma eggs and plays an important role in fibrogenesis

\section{A) Murine Schistosomiasis}
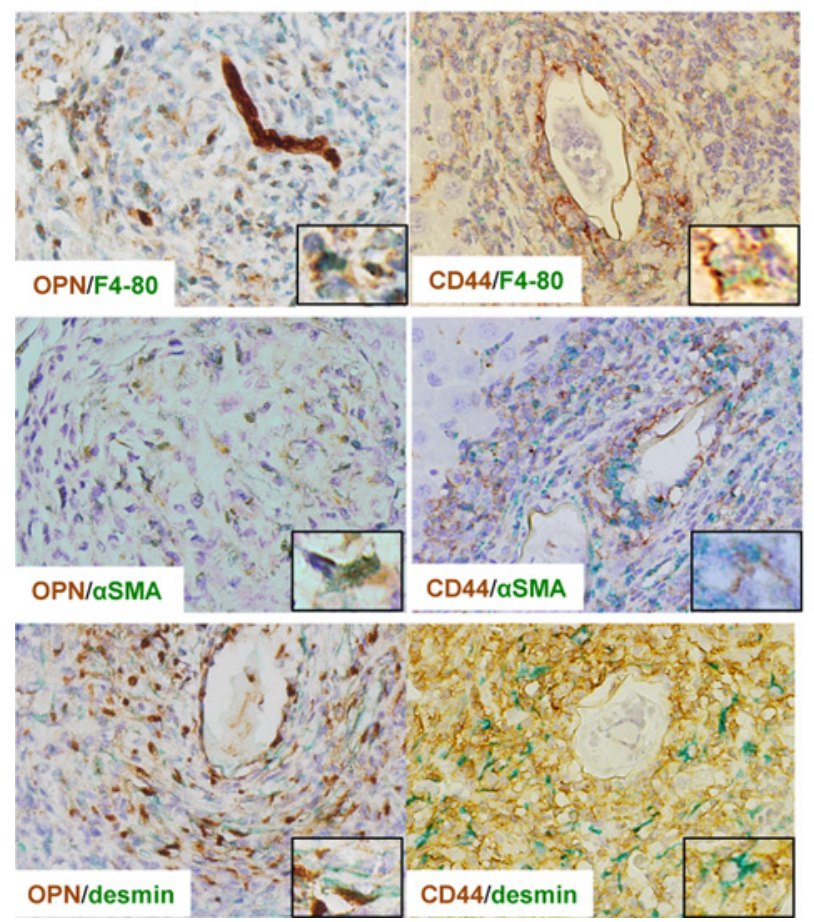

\section{B) Human Schistosomiasis}
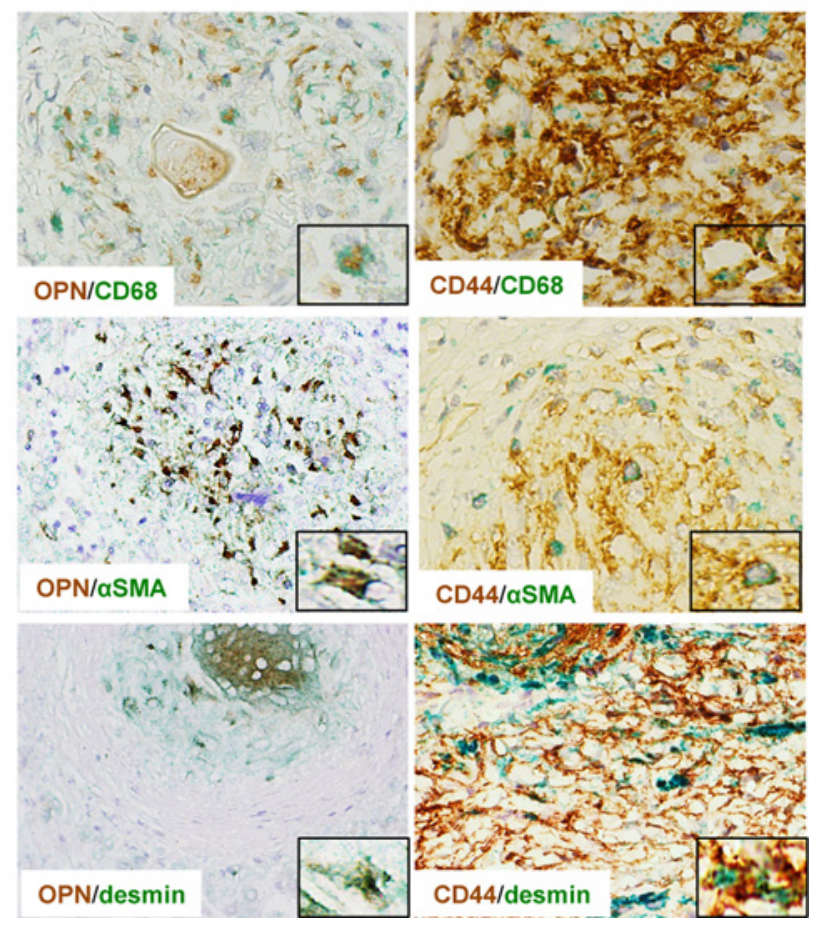

Figure 5 Macrophages and myofibroblasts/HSCs produce and respond to OPN in schistosomiasis

Double immunostaining for OPN (left panels) or one of its receptors (CD44; right panels) and a macrophage marker (F4-80 for mice, CD68 for humans) or myofibroblast/HSC markers ( $\alpha$ SMA and desmin) in murine (A) and human (B) schistosomiasis mansoni, showing that those cells produce and respond to OPN (final magnifications: $\times 400$, inset $\times 1000$ ). 
because reactive ductular cells are a major source of the profibrogenic molecule OPN.

Recent studies found that cholangiocyte-derived OPN both promotes the ductular reaction and acts in a paracrine fashion to stimulate HSC to become fibrogenic myofibroblasts $[22,30]$. These observations support our current findings that OPN expression in bile ducts correlates with myofibroblast accumulation and fibrosis severity in mice and humans chronically infected with $S$. mansoni. The aggregate results suggest that OPN may be a novel target for anti-fibrotic therapy, which would help to reduce life-threatening complications from this tropically endemic infectious disease. Indeed, evidence supporting a causal role for OPN in schistosoma-related fibrogenesis was previously described in a study of Schistosoma japonica-infected mice $[19,20]$. In that study, OPN neutralization decreased myofibroblast activation and reduced collagen deposition [20]. However, the work did not identify which cells produced or responded to OPN in the infected mice and humans with schistosomiasis japonica were not studied. The present study is the first to investigate OPN in schistosomiasis mansoni and it includes human data, confirming that this molecule plays a pivotal and clinically relevant role in schistosomiasis. Our work also demonstrates, for the first time, that circulating OPN levels correlate with fibrosis severity in murine and human schistosomiasis. This finding is noteworthy because it supports the use of OPN as a potential biomarker for the severity of schistosomal liver disease.

Finally, we present the first evidence that links OPN with portal hypertension in patients with chronic schistosomal infection. This is clinically relevant and noteworthy because morbidity and mortality result mainly from complications of portal hypertension in this disease [6]. Previously, another group had reported that OPN levels were higher in biliary atresia patients with portal hypertension than in biliary atresia patients without portal hypertension [16]. Our findings extend that initial observation by documenting that OPN levels directly correlate with portal pressures. This novel finding has profound implications because it suggests that OPN not only regulates hepatic fibrogenesis, but also reflects the severity of portal hypertension and the latter drives morbidity and mortality in most liver diseases. Additional studies will be necessary to confirm its use as a possible morbidity biomarker.

In conclusion, $S$. mansoni egg antigens stimulate cholangiocyte proliferation and OPN secretion. OPN levels in the liver and blood correlate with fibrosis stage and severity of portal hypertension.

\section{CLINICAL PERSPECTIVES}

- Schistosomiasis mansoni is a major cause of fibrosis and portal hypertension worldwide. The mechanisms driving this tropical disease remain poorly understood, despite long-standing evidence that granuloma-associated factors drive the process. This gap in knowledge has limited the development of both effective treatments to inhibit/reverse fibrosis and non-invasive biomarkers.
- In the present study, we demonstrated, for the first time, that schistosomal egg antigens directly induce host cholangiocytes to proliferate and produce the pro-fibrogenic molecule OPN. Serum/plasma and hepatic OPN levels not only correlate with liver myofibroblast accumulation and liver fibrosis stage, but strictly match the splenic venous pressure, a direct measure of portal hypertension.

- OPN is a novel therapy target candidate to treat liver fibrosis and portal hypertension and a possible non-invasive biomarker in schistosomiasis mansoni.

\begin{abstract}
AUTHOR CONTRIBUTION
Thiago Pereira, Wing-Kin Syn, Anna Diehl, José Lambertucci, Zilton Andrade and Paula Vidigal conceived and designed the experiments. Thiago Pereira, Mariana Machado, Elisângela Santos, Márcia Souza, Vivian Resende, Guanhua Xie and Isaac Chan performed the experiments. Thiago Pereira, Anna Diehl, Wing-Kin Syn, José Lambertucci and Fausto Pereira analysed the data. Izabela Voieta, Paula Vidigal, Vivian Resende, Alba Otoni and Guilherme Trindade contributed with acquisition of clinical data and human samples. Fausto Pereira, José Lambertucci, Anna Diehl, William Secor, Rafal Witek, Zilton Andrade and Steve Choi contributed with reagents/materials/analysis tools. Thiago Pereira, Wing-Kin Syn and Anna Diehl wrote the paper.
\end{abstract}

\section{ACKNOWLEDGEMENTS}

We thank Mr Carl Stone for administrative support and Mr Antônio Carlos Silva Santos for technical support.

\section{FUNDING}

This work was support by the National Institutes of Health [grant number R01-DK-077794 (to A.M.D.)]; the Duke University Endowment [the Florence McAlister Professorship (to A.M.D.)]; the Federal Council for Research and Development (to F.E.P., J.R.L., T.A.P. and Z.A.A.); and the Fundação de Amparo à Pesquisa do Estado de Minas Gerais (FAPEMIG) (to J.R.L.).

\section{REFERENCES}

1 Colley, D.G., Bustinduy, A.L., Secor, W.E. and King, C.H. (2014) Human schistosomiasis. Lancet 383, 2253-2264 CrossRef PubMed

2 Chitsulo, L., Engels, D., Montresor, A. and Savioli, L. (2000) The global status of schistosomiasis and its control. Acta Trop. 77, 41-51 CrossRef PubMed

3 Gryseels, B., Polman, K., Clerinx, J. and Kestens, L. (2006) Human schistosomiasis. Lancet 368, 1106-1118 CrossRef PubMed

4 Andrade, Z.A. (2009) Schistosomiasis and liver fibrosis. Parasite Immunol. 31, 656-663 CrossRef PubMed

5 Andrade, Z.A. (2004) Schistosomal hepatopathy. Mem. Inst. Oswaldo Cruz 99 (Suppl. 1), 51-57 CrossRef PubMed

6 Lambertucci, J.R. (2014) Revisiting the concept of hepatosplenic schistosomiasis and its challenges using traditional and new tools. Rev. Soc. Bras. Med. Trop. 47, 130-136 CrossRef PubMed 
7 Burke, M.L., Jones, M.K., Gobert, G.N., Li, Y.S., Ellis, M.K. and McManus, D.P. (2009) Immunopathogenesis of human schistosomiasis. Parasite Immunol. 31, 163-176 CrossRef PubMed

8 Pereira, T.A., Xie, G., Choi, S.S., Syn, W.K., Voieta, I., Lu, J., Chan, I.S., Swiderska, M., Amaral, K.B., Antunes, C.M. et al. (2013) Macrophage-derived Hedgehog ligands promotes fibrogenic and angiogenic responses in human schistosomiasis mansoni. Liver Int. 33, 149-161 CrossRef PubMed

9 Omenetti, A. and Diehl, A.M. (2011) Hedgehog signaling in cholangiocytes. Curr. Opin. Gastroenterol. 27, 268-275 CrossRef PubMed

10 Syn, W.K., Choi, S.S., Liaskou, E., Karaca, G.F., Agboola, K.M., Oo, Y.H., Mi, Z., Pereira, T.A., Zdanowicz, M., Malladi, P. et al. (2011) Osteopontin is induced by hedgehog pathway activation and promotes fibrosis progression in nonalcoholic steatohepatitis. Hepatology 53, 106-115 CrossRef PubMed

11 Morales-lbanez, O., Dominguez, M., Ki, S.H., Marcos, M., Chaves, J.F., Nguyen-Khac, E., Houchi, H., Affo, S., Sancho-Bru, P., Altamirano, J. et al. (2013) Human and experimental evidence supporting a role for osteopontin in alcoholic hepatitis. Hepatology 58, 1742-1756 CrossRef PubMed

12 Patouraux, S., Bonnafous, S., Voican, C.S., Anty, R., Saint-Paul, M.C., Rosenthal-Allieri, M.A., Agostini, H., Njike, M., Barri-Ova, N., Naveau, S. et al. (2012) The osteopontin level in liver, adipose tissue and serum is correlated with fibrosis in patients with alcoholic liver disease. PLoS One 7, e35612 CrossRef PubMed

13 Syn, W.K., Agboola, K.M., Swiderska, M., Michelotti, G.A., Liaskou, E., Pang, H., Xie, G., Philips, G., Chan, I.S., Karaca, G.F. et al. (2012) NKT-associated hedgehog and osteopontin drive fibrogenesis in non-alcoholic fatty liver disease. Gut $6 \mathbf{1}$, 1323-1329 CrossRef PubMed

14 Zhao, L., Li, T., Wang, Y., Pan, Y., Ning, H., Hui, X., Xie, H., Wang, J., Han, Y., Liu, Z. and Fan, D. (2008) Elevated plasma osteopontin level is predictive of cirrhosis in patients with hepatitis B infection. Int. J. Clin. Pract. 62, 1056-1062 CrossRef PubMed

15 Choi, S.S., Claridge, L.C., Jhaveri, R., Swiderska-Syn, M., Clark, P., Suzuki, A., Pereira, T.A., Mi, Z., Kuo, P.C., Guy, C.D. et al. (2014) Osteopontin is up-regulated in chronic hepatitis $C$ and is associated with cellular permissiveness for hepatitis $C$ virus replication. Clin. Sci. 126, 845-855 CrossRef PubMed

16 Huang, L., Wei, M.F. and Feng, J.X. (2008) Abnormal activation of OPN inflammation pathway in livers of children with biliary atresia and relationship to hepatic fibrosis. Eur. J. Pediatr. Surg. 18, 224-229 CrossRef PubMed

17 Shang, S., Plymoth, A., Ge, S., Feng, Z., Rosen, H.R., Sangrajrang, S., Hainaut, P., Marrero, J.A. and Beretta, L. (2012) Identification of osteopontin as a novel marker for early hepatocellular carcinoma. Hepatology 55, 483-490 CrossRef PubMed

18 O’Regan, A.W., Hayden, J.M., Body, S., Liaw, L., Mulligan, N., Goetschkes, M. and Berman, J.S. (2001) Abnormal pulmonary granuloma formation in osteopontin-deficient mice. Am. J. Respir. Crit. Care Med. 164, 2243-2247 CrossRef PubMed
19 Chen, B.L., Zhang, G.Y., Yuan, W.J., Wang, S.P., Shen, Y.M., Yan, L., Gu, H. and Li, J. (2011) Osteopontin expression is associated with hepatopathologic changes in Schistosoma japonicum infected mice. World J. Gastroenterol. 17, 5075-5082 CrossRef PubMed

20 Chen, B.L., Zhang, G.Y., Wang, S.P., Li, Q., Xu, M.H., Shen, Y.M., Yan, L., Gu, H., Li, J., Huang, Y.L. and Mu, Y.B. (2012) The combined treatment of praziquantel with osteopontin immunoneutralization reduces liver damage in Schistosoma japonicum-infected mice. Parasitology 139, 522-529 CrossRef PubMed

21 Ramaiah, S.K. and Rittling, S. (2008) Pathophysiological role of osteopontin in hepatic inflammation, toxicity, and cancer. Toxicol. Sci. 103, 4-13 CrossRef PubMed

22 Coombes, J.D., Swiderska-Syn, M., Dolle, L., Reid, D., Eksteen, B., Claridge, L., Briones-Orta, M.A., Shetty, S., Oo, Y.H., Riva, A. et al. (2015) Osteopontin neutralisation abrogates the liver progenitor cell response and fibrogenesis in mice. Gut 64, 1120-1131 PubMed

23 Nagoshi, S. (2014) Osteopontin: versatile modulator of liver diseases. Hepatol. Res. 44, 22-30 CrossRef PubMed

24 Omenetti, A., Syn, W.K., Jung, Y., Francis, H., Porrello, A., Witek, R.P., Choi, S.S., Yang, L., Mayo, M.J., Gershwin, M.E. et al. (2009) Repair-related activation of hedgehog signaling promotes cholangiocyte chemokine production. Hepatology 50, 518-527 CrossRef PubMed

25 Omenetti, A., Porrello, A., Jung, Y., Yang, L., Popov, Y., Choi, S.S. Witek, R.P., Alpini, G., Venter, J., Vandongen, H.M. et al. (2008) Hedgehog signaling regulates epithelial-mesenchymal transition during biliary fibrosis in rodents and humans. J. Clin. Invest. 118, 3331-3342 PubMed

26 da Silva, L.C., Alves, V.A., Abrantes, C.P., Lima, D.M., Christo, C.H. and De Brito, T. (1985) Effects of chemotherapy on mice submitted to multiple Schistosoma mansoni infections. A controlled randomized prospective study. Trop. Med. Parasitol. 36, 150-154 PubMed

27 da Silva, L.C., Vianna, M.R., Abrantes, C.P., Lima, D.M., Falavigna, A.L., Antonelli-Cardoso, R.H., Gallucci, S.D. and de Brito, T. (1990) Liver morphology with emphasis on bile ducts changes and survival analysis in mice submitted to multiple Schistosoma mansoni infections and chemotherapy. Rev. Inst. Med. Trop. Sao Paulo 32, 328-337

CrossRef PubMed

28 Oliveira, L. and Andrade, Z.A. (2005) Significance of bile-duct changes in schistosomiasis. Rev. Soc. Bras. Med. Trop. 38, 464-468 CrossRef PubMed

29 Vianna, M.R., Gayotto, L.C., Telma, R., Santos, M., Alves, V.A., Fukushima, J. and de Brito, T. (1989) Intrahepatic bile duct changes in human hepatosplenic schistosomiasis mansoni. Liver 9, 100-109 CrossRef PubMed

30 Wang, X., Lopategi, A., Ge, X., Lu, Y., Kitamura, N., Urtasun, R., Leung, T.M., Fiel, M.I. and Nieto, N. (2014) Osteopontin induces ductular reaction contributing to liver fibrosis. Gut $\mathbf{6 3}$, 1805-1818 CrossRef PubMed 